TMS bursts can modulate local and networks oscillations during lower-limb movement

\author{
Arturo I. Espinoza ${ }^{+, \wedge}$, Jamie L. Scholl ${ }^{*++,}$, Arun Singh ${ }^{*,++, \#}$ \\ ${ }^{+}$Neurology Department, University of Iowa, Iowa City, IA, USA \\ ${ }^{*}$ Center for Brain and Behavior Research, University of South Dakota, Vermillion, SD, USA \\ ${ }^{++}$Division of Basic Biomedical Sciences, Sanford School of Medicine, University of South \\ Dakota, Vermillion, SD, USA
}

${ }^{\wedge}$ These authors contributed equally.

\title{
\#Correspondence to:
}

Dr. Arun Singh

Division of Basic Biological Sciences, Sanford School of Medicine, University of South Dakota, 414 E. Clark St. Vermillion, SD, 57069, USA

Email: arun.singh@usd.edu

Key Words: TMS bursts; EEG; oscillations; lower-limb movement; theta-band. 


\begin{abstract}
Introduction: Lower-limb motor functions involve processing information via both motor and cognitive control networks. Measuring oscillations is a key element in communication within and between cortical networks during high order motor functions. Increased midfrontal theta oscillations are related to improved lower-limb motor performances in patients with movement disorders. Non-invasive neuromodulation approaches have not been explored extensively to understand the oscillatory mechanism of lower-limb motor functions. This study aims to examine the effects of repetitive transcranial magnetic stimulation (rTMS) on local and network EEG oscillations in healthy elderly subjects.
\end{abstract}

Methods: Eleven healthy elder subjects (67-73 years) were recruited via advertisements, and underwent both active and sham stimulation procedures in a random, counterbalanced design. TMS bursts $(\theta$-TMS; 4 pulses/sec) were applied over the midfrontal lead (vertex) before a GOCue pedaling task, and signals were analyzed using time-frequency methods.

Results: TMS bursts increase the theta activity in the local $(p=0.02)$, as well as the associated network during the lower-limb pedaling task $(\mathrm{p}=0.02)$. Furthermore, after task-related TMS burst sessions, increased resting-state alpha activity was observed in the midfrontal region $(\mathrm{p}=0.01)$.

Conclusion: Our study suggests the ability of midfrontal TMS bursts to directly modulate local and network oscillations in a frequency manner during lower-limb motor task. TMS burst-induced modulation may provide insights into the functional roles of oscillatory activity during lower-limb movement in normal and disease conditions. 


\section{INTRODUCTION}

Increasing evidence has shown the role of cortical oscillations in conveying information within motor and cognitive control networks. ${ }^{1-3}$ Within the motor and cognitive research field, midfrontal theta, beta, and gamma oscillations have been associated with motor tasks with cognitive recruitment such as gait or lower-limb movements. ${ }^{4-6}$ Cognitive contributions to lowerlimb movement in elderly people ( $>50$ years old) and patients with movement disorders are wellacknowledged in movement sciences. ${ }^{7,8}$ Additionally, the role of midfrontal theta oscillations in cognitive control has been studied extensively in neuropsychiatric and movement disorders patients. ${ }^{3,9}$ Animal studies have shown increased theta oscillations around the anterior cingulate cortex and frontal cortex, similar to frontal midline theta oscillations in humans, during attentional motor tasks. ${ }^{10,11}$ These studies have spurred a growing interest in the manipulation of midfrontal theta oscillations to improve attentional motor tasks. A better understanding of their causal physiological role can be achieved in the coding of movements involving attentional processes. Indeed, this endeavor has the potential to alleviate lower-limb motor problems in patients with movement disorders.

Non-invasive cortical stimulation techniques such as transcranial magnetic and electrical stimulation (TMS and tES) have emerged as promising neuromodulation methods, enabling direct modulation of oscillations in the local and related networks. ${ }^{12,13}$ TMS, specifically repetitive TMS (rTMS), appears to be a particularly effective neuromodulation tool to target spatially confined cortical regions, and in modulating oscillations in the local region and associated neural networks. ${ }^{14,15}$ In comparison to a single pulse, TMS bursts can induce long-term plastic changes in the motor and cognitive networks and modulate neuronal oscillations effectively, leading to an increased interest in therapeutic possibilities. ${ }^{16,17}$ Recent findings have shown that TMS bursts in 
the motor cortical region can improve gait and balance problems in Parkinson's Disease (PD) patients $^{18,19}$, however, the mechanisms by which TMS bursts exert neuromodulatory effects on lower-limb motor performance remain unknown.

TMS's ability to modulate motor networks in combination with EEG's ability to record changes in oscillatory network activity with high temporal resolution makes it an ideal method to understand underlying motor performance. ${ }^{20,21}$ Nevertheless, it remains unclear whether TMS bursts ( $\theta$-TMS; 4 arrhythmic pulses in a second) result in entrainment for theta frequency bands in the local stimulated brain region. Furthermore, it is not known how locally modulated oscillations impact resultant activity in the associated cortical regions during lower-limb movement in an elderly population. To address these questions, we analyzed EEG recordings in healthy elderly subjects, while applying TMS bursts to the midfrontal region (at vertex). The motor and cognitive deficits thought to be related to theta oscillations are typically found in a patient population such as $\mathrm{PD} .^{3,6}$ As the average age of initial onset for PD is 60 , baseline network activity was determined in a non-patient population within a similar age group, to better understand how these networks are affected in a comparable control group, prior to examining them in a patient population with network dysfunction.

\section{METHODS}

\section{PARTICIPANTS}

Eleven healthy elderly subjects (mean \pm sem; age $70 \pm 3$ years; $6 \mathrm{~F} / 5 \mathrm{M}$ ) were recruited in Iowa via advertisements to participate in the study. All protocols were approved by the University of South Dakota Institutional Review Board and the University of Iowa Institutional Review Board in accordance with the Declaration of Helsinki. Recruitment was limited to older adults, as a healthy, age-appropriate control group for future PD patient population studies. All subjects were 
free from orthopedic conditions, and were screened for exclusionary criteria, using our laboratory's standard questionnaire, which consists of neurological and mental health history, psychoactive medication use, presence of a cardiac pacemaker or any other metal implant. All participants provided written informed consent to participate in the study.

\section{LOWER-LIMB PEDALING MOTOR TASK AND ANALYSIS}

Similar to our previous study, ${ }^{6}$ a pedaling motor task was used to study lower-limb motor control. Participants were instructed to start pedaling upon seeing the GO-cue and to stop the pedals in the starting position after one rotation until the next GO-cue. This type of motor task induces minimal movement-related artifact and requires bilateral lower-limb coordination similar to gait. Furthermore, this paradigm can be used to investigate feedback-based neuromodulation. The pedaling task involves 2 blocks, each consisting of 25 trials during which the subject remains seated. Fifty total trials provide adequate signal-to-noise ratios for subsequent EEG analysis, and also prevents fatigue in elderly participants as demonstrated in our EEG work. ${ }^{6,22}$ For each trial, a warning cue briefly appeared on a stimulus presentation computer screen. Afterwards, a TMS burst was delivered to the midfrontal region $(\mathrm{Cz})$ before the GO-Cue, triggered by TTL input. The $2 \mathrm{~s}$ GO-Cue appeared $3 \pm 0.6 \mathrm{~s}$ after the $4^{\text {th }}$ TMS pulse discharge (Fig. 1A), with an inter-trial interval of $3 \pm 0.1 \mathrm{~s}$.

The total duration of each pedaling trial averaged $10.4 \pm 0.7 \mathrm{~s}$ across subjects. A 3-axis accelerometer was attached to the subject's left ankle. Afterwards, all three acceleration signals were averaged to generate a single signal. Linear speed for each trial was analyzed in single signal during the 0-2 s time window following the GO-Cue signal, as participants took approximately 2 
s to complete one pedal rotation. Signals were detrended, and a $5 \mathrm{~Hz}$ low-pass filter was applied prior to computing the linear speed. Mean linear speed for each subject $(\mathrm{m} / \mathrm{s})$ was calculated.

\section{ACTIVE AND SHAM TMS TO THE MIDFRONTAL REGION}

We used a figure-of-eight TMS coil (MC-B70) connected to a MagVenture (MagVenture, Inc., Alpharetta GA) Stimulator (MagPro X100) in both active and sham stimulation conditions over the midfrontal ("Cz" vertex) region according to the EEG 10/20 electrode position. We selected the midfrontal location for the TMS to modulate theta oscillations in the target and connected regions. ${ }^{3,6}$ Previous studies have demonstrated that stimulation over the vertex can increase leg motor cortex excitability bilaterally, and induce the most stable responses in the leg motor area. ${ }^{23,24}$ The current study followed the suggested TMS methodological checklist to improve the quality of data collection and reporting results. ${ }^{25}$ For sham stimulation, the figure-ofeight coil was inverted relative to the target location and off-targeted, which reduced the magnetic field significantly to below the threshold necessary to modulate oscillations. ${ }^{26,27}$ Stimulation intensity was set at $90 \%$ of the individuals resting motor threshold (RMT). RMT was determined on each subject, and is the minimum intensity that elicited at least five finger twitches in response to ten consecutive single-pulses applied to the motor hot spot.

Participants were blinded to stimulation, and both active and sham stimulation conditions were counterbalanced and randomized, with a minimum of 60 min between sessions; which has been shown to exceed the response time for the modulatory effects of theta burst TMS on cognitive tasks. ${ }^{28}$ A single TMS burst ( $\theta$-TMS) was applied, which consisted of 4 consecutive arrhythmic biphasic pulses within $1 \mathrm{~s}$, and onset of TMS pulses differed for each trial and participant. 


\section{EEG-TMS RECORDING AND PREPROCESSING}

Scalp EEG-TMS signals were collected during the lower-limb pedaling task using a 64-

channel EEG actiCAP (Brain Products GmbH, Gilching, Germany) and pycorder software with high-pass anti-aliasing FIR filter of $0.1 \mathrm{~Hz}$ with order 1 and $10 \mathrm{kHz}$ sampling frequency. EEG signals were also collected for 2-3 min during the eyes-open resting-state condition after completing pedaling task.

Reference and grounded channels were electrodes Pz and Fpz, respectively. Channels that were considered unreliable due to muscle artifacts or white noise (Fp1, Fp2, FT10, TP9, and TP10) were removed. EEG signals were epoched around the GO-Cue (-0.6 s to 2.4 s) to exclude TMSevoked artifacts occurring prior to GO-Cue. Epochs with excessively noisy EEG or muscle artifacts were excluded using a conjunction of the Fully Automated Statistical Thresholding for EEG artifact Rejection (FASTER) algorithm with default parameters ${ }^{29}$ and pop_rejchan from EEGLAB. ${ }^{30}$ Eye movement artifacts were removed using an automatic EEG artifact detection algorithm based on the joint use of spatial and temporal features (ADJUST) with default parameters. ${ }^{31}$ Subsequently, data was rereferenced to common average reference.

\section{EEG POSTPROCESSING ANALYSIS}

Preprocessed (epoched) signals were used for time-frequency analysis by complex Morlet wavelets, as explained previously. ${ }^{3,6,32}$ Further, epochs were cut in -0.5 to $+2.0 \mathrm{~s}$ windows, frequency bands between 1 and $50 \mathrm{~Hz}$ in logarithmically-spaced bins were chosen, and power was normalized by conversion to a decibel $(\mathrm{dB})$ scale. Similar to prior studies, the baseline for each frequency consisted of the average power from -0.3 to $-0.2 \mathrm{~s}$ prior to the onset of the GO-Cue., ${ }^{6,32}$ Time-frequency analyses was limited to electrode $\mathrm{Cz}$, and a-priori time-frequency Regions of 
Interest (tf-ROI). tf-ROIs were preselected for the theta-band $(3-7 \mathrm{~Hz})$ and the time window of interest was +0.5 to $+1.5 \mathrm{~s}$ following the GO-Cue. tf-ROIs mean power values were used for statistical comparisons, with the main analysis restricted on a-priori tf-ROI as it represents the specificity, and tf-ROIs topographic map was plotted and compared between active versus sham conditions.

Preprocessed signals were also used for time-frequency spectral coherence (magnitudesquared coherence) analysis. A -0.3 to $-0.2 \mathrm{~s}$ window was used as a baseline to compute change in coherence, then spectral coherence algorithms were implemented as explained in detail by Cohen. ${ }^{33}$ Spectral coherence over trials between $\mathrm{Fz}$ and $\mathrm{Cz}$ (fronto-cortical network) and $\mathrm{C} 3$ and C4 (inter-hemispheric motor cortical network) was computed. The same priori tf-ROIs were used (as above) to export coherence values to compare active versus sham stimulation data.

EEG resting-state signals were preprocessed (similar to above) and Welch's power spectral density estimate (pwelch) Matlab function was applied on epoched (3.5 s) signals to compute spectral properties. Relative power at delta and alpha frequency bands was quantified compared to the power over $0.1-50 \mathrm{~Hz}$. The resting-state mean relative power, after the task-related active and sham TMS bursts, was compared. Resting-state analysis was focused on the Cz electrode.

Wilcoxon signed rank tests were used to compare behavior and electrophysiological data between active TMS and sham. Effect sizes were computed as Cohen's d. Statistical analysis was focused on our a-priori hypothesis to determine the specificity and restricted to midfrontal (“Cz") electrode and theta-band activity only. In addition to this, we employed threshold-based correction on the full time-frequency plots in order to reveal any other reliable differences between active and sham TMS, and these differences are shown as black contour in time-frequency plots. The 
alpha level was 0.05 and Bonferroni adjustments were applied with adjusted alpha level 0.026 where appropriate.

\section{RESULTS}

Active TMS bursts ( $\theta$-TMS) significantly increased the theta-band power when compared to a within-subjects sham stimulation during pedaling task in healthy elderly subjects $(\mathrm{z}=-2.31, \mathrm{p}$ $=0.02$, Cohen's $d=-0.934$, Fig. 1B-E, see Fig. S1 for each subject). Time-frequency plots show no changes in the other frequency bands power (Fig. 1B-E). Our results distinctly demonstrate that $\theta$-TMS bursts ( 4 pulses/sec) can enhance the power in the burst frequency which is the theta range in our study.

Spectral coherence analysis between frontal leads $(\mathrm{Fz}-\mathrm{Cz})$ showed increased pedaling-task related coherence within the theta frequency range after active TMS (Fig. 2A-C, p $<0.05$, see the black contour in figure 2C). A significant increase in coherence in the tf-ROI theta-band was observed after active $\theta$-TMS bursts as compared to sham $(z=-2.2, p=0.026$, Cohen's $d=-0.70$, Fig. 2D). Furthermore, we analyzed spectral coherence between left and right motor cortical leads (C3-C4) to observe coherent activity in the inter-hemispheric motor cortical network. Interhemispheric motor cortical network showed increased pedaling-task related coherence within the theta frequency range after active $\theta$-TMS bursts (Fig. S2A-C; $<<0.05$, see the black contour). A significant increase in coherence in the theta-band was also seen in the inter-hemispheric motor cortical network after active $\theta$-TMS bursts as compared to sham $(z=-2.04, p=0.04$, Cohen's $d=$ -0.65 , Fig. S2D). In line with previous reports, our results also suggest that TMS bursts may modulate the cortical network oscillations during lower-limb motor task. ${ }^{15,34}$

Further, we analyzed poststimulation resting state signals to investigate the overall changes in the spectral properties after the active and sham $\theta$-TMS burst sessions (Fig. 3A). No significant 
difference in relative power was found in the theta-band in the midfrontal lead after active TMS burst sessions compared to sham $(z=1.96, p=0.05$, Cohen's $d=0.66$, Fig. 3B). However, we found decrease in relative power in the alpha-band $(z=2.58, p=0.01$, Cohen's $d=0.91$, Fig. 3C) in the midfrontal lead. In line with current results, previous studies have also reported that following transcranial stimulation protocols, changes in resting-state alpha oscillations have been shown to modulate cognitive functions. ${ }^{20,35,36}$.

Our data showed an increase in acceleration (Fig. 4B) and a time-dependent increase in pedaling speed following active $\theta$-TMS bursts when compared to sham (Fig. 4C). However, we did not observe significant change in the pedaling speed in our subjects across trials (mean \pm sem speed: sham $=0.2 \pm 0.01 \mathrm{~m} / \mathrm{s} ;$ active $=0.3 \pm 0.04 \mathrm{~m} / \mathrm{s} ; \mathrm{z}=0.8, \mathrm{p}=0.42$, Cohen's $\mathrm{d}=-0.23$ )

\section{DISCUSSION}

To investigate the immediate effects of TMS bursts on a lower-limb motor task, it is crucial to identify the actions of TMS on local and network oscillations. Our results suggest that TMS can modulate brain oscillations in direct interaction with the underlying generator, via synchronized midfrontal lower-limb task-related theta activity when targeting the theta $(4-7 \mathrm{~Hz})$ oscillations generator. We also demonstrate that $\theta$-TMS bursts can enhance theta oscillations in the associated network. Results suggest that $\theta$-TMS bursts may enhance the naturally occurring theta oscillations rather than impose artificial oscillations. The overall data support the hypothesis that TMS bursts may drive natural brain oscillations by entrainment, and this might be a possible mechanism via which TMS may act on the local and associated oscillatory activities to influence motor behavior. ${ }^{20,34,37}$

Movement disorder patients with lower-extremity dysfunctions have shown impaired cognitive control and decreased frontal theta oscillations, and thus show impaired kinematics of 
lower-limb movement ${ }^{6}$. Attention is an important factor when performing lower-limb movements, especially when walking under dual-task conditions. ${ }^{8,38}$ Midfrontal theta oscillations may play the primary role in providing strong cognitive engagement during dual-tasks, ${ }^{6,39}$ contributing to the sensorimotor integration needed to perform lower-limb motor tasks. The current task-related EEG data reveal that $\theta$-TMS bursts can upregulate targeted theta oscillations in higher-order frontal regions of the attention network and may enhance lower-limb movements.

Frontal beta oscillations also play a critical role in the top-down pathway conveying the information from preparatory and execution plans during lower-limb motor tasks, and these processes may malfunction in PD patients and other movement disorders. ${ }^{2,40}$ Increased beta-band power in the cortical and basal ganglia networks are associated with motor abnormalities in patients with PD. ${ }^{2,41}$ The dopamine precursor, levodopa, has shown the modulatory effects on the cortical and sub-cortical beta oscillations as well as the degree of motor impairment in patients with PD. ${ }^{42,43}$ The existence of two principal modes of synchronized oscillations within the human subthalamo-pallidal-thalamo-cortical circuit, at $<30 \mathrm{~Hz}$ has been suggested in PD, and these oscillations are systematically modulated by motor task, thereby suggesting a functional role in movement. ${ }^{44}$ It has been shown that invasive deep brain stimulation (DBS) attenuates the betaband activity and improves upper-limb movement in PD patients. ${ }^{45,46}$ However, fewer DBS studies have shown a therapeutic option for improving lower-limb movements such as gait in patients with PD. ${ }^{47,48}$ Similar to invasive studies, non-invasive TMS bursts approach may decrease cortical betaband power and improve gait or lower-limb performances in PD. ${ }^{19}$ The overall data may support our previous hypothesis that increased frontal theta activity and reduced beta activity are associated with improvement in lower-limb movement in PD patients with gait abnormalities. ${ }^{6}$ 
High order lower-limb movements are a network phenomenon; and aberrations in the cortico-cortical and motor cortical networks have been observed in PD patients with gait abnormalities. ${ }^{2,49}$ Non-invasive TMS protocols can modulate oscillations in the target and associated networks during motor and cognitive tasks. ${ }^{19,50}$ In line with these studies, current results demonstrate the capacity of TMS bursts protocol to modulate theta oscillations in the corticocortical and motor cortical networks, which may be correlated with changes in the motor behavior even with the cognitive load. While published reports indicate that TMS bursts can improve motor performance in patients with movement disorders, ${ }^{19,51}$ we did not see a change in motor function after stimulation. Most likely, absent motor findings in our study reflect the low elderly subject numbers. However, uncoupling of EEG and motor effects of repetitive TMS is also a possibility.

Additionally, results show that $\theta$-TMS bursts did not modulate resting-state theta oscillations, suggesting the change in theta activity is most-likely associated with GO-Cuetriggered motor performance. ${ }^{3,6}$ However, an increase in resting-state alpha-band activity following TMS bursts sessions demonstrates the overall poststimulation effect in the target region, which may also be related to improvement in motor and cognitive control systems. ${ }^{20,36}$ Notably, changes in alpha oscillations have been observed after transcranial neuromodulation in previous human studies, ${ }^{35,52}$ and these oscillations have been implicated in top-down cortical processing. ${ }^{53}$

The limitations of this study include: 1) Behavioral non-responders in the pedaling task, perhaps due to small participant numbers or the application of $90 \%$ RMT being insufficient to induce changes in pedaling speed, however, strong modulation in the theta-band activity was observed; 2) TMS was applied over the midfrontal region rather than specifically over the leg motor cortical area, however, current methods have been shown to induce leg motor cortex excitability in both hemispheres ${ }^{23,24}$ In addition, TMS over midfrontal region can improve 
cognitive processing; 3) Use of a small, non-patient target population to explore the underlying mechanisms in the control of lower-limb motor networks prior to testing in a patient population rather than including the patient population in the experimental design. Nonetheless, data generated with this group will serve as a control for the age range in the patient population that will be examined in future studies.

\section{CONCLUSIONS}

The current study may provide an efficient, non-invasive method to normalize brain oscillations that are often abnormal in conditions with motor disabilities. Specifically, by combining the TMS bursts protocol utilized with specific motor paradigms, this approach has the potential to investigate the causal links between midfrontal brain oscillations and lower-limb movement in humans. ${ }^{6}$ Further, this method may provide an understanding of the neurophysiological basis of modulations in the local and associated oscillatory networks, potentially contributing to novel rehabilitation regimens in patient populations such as PD.

\section{ACKNOWLEDGEMENTS}

The authors thank all participants in this study. AIE is supported by the University of Iowa, IA. JLS and AS are supported by the Division of Basic Biomedical Sciences, University of South Dakota, Vermillion, SD, USA. We also thank Dr. Lee A. Baugh for his efforts towards improving our manuscript. 


\section{REFERENCES}

1. Buzsaki G, Draguhn A. Neuronal oscillations in cortical networks. Science 2004;304:19261929.

2. Singh A. Oscillatory activity in the cortico-basal ganglia-thalamic neural circuits in Parkinson's disease. Eur J Neurosci 2018;48:2869-2878.

3. Singh A, Richardson SP, Narayanan N, Cavanagh JF. Mid-frontal theta activity is diminished during cognitive control in Parkinson's disease. Neuropsychologia 2018;117:113-122.

4. Peterson SM, Ferris DP. Differentiation in Theta and Beta Electrocortical Activity between Visual and Physical Perturbations to Walking and Standing Balance. eNeuro 2018;5.

5. Seeber M, Scherer R, Wagner J, Solis-Escalante T, Müller-Putz GR. EEG beta suppression and low gamma modulation are different elements of human upright walking. Front Hum Neurosci 2014;8.

6. Singh A, Cole RC, Espinoza AI, Brown D, Cavanagh JF, Narayanan NS. Frontal theta and beta oscillations during lower-limb movement in Parkinson's disease. Clin Neurophysiol 2020;131:694-702.

7. Eggermont LH, Gavett BE, Volkers KM, et al. Lower-extremity function in cognitively healthy aging, mild cognitive impairment, and Alzheimer's disease. Arch Phys Med Rehabil 2010;91:584-588.

8. Li KZH, Bherer L, Mirelman A, Maidan I, Hausdorff JM. Cognitive Involvement in Balance, Gait and Dual-Tasking in Aging: A Focused Review From a Neuroscience of Aging Perspective. Front Neurol 2018;9.

9. Cavanagh JF, Frank MJ. Frontal theta as a mechanism for cognitive control. Trends Cogn Sci 2014;18:414-421.

10. Tsujimoto T, Shimazu H, Isomura Y. Direct Recording of Theta Oscillations in Primate Prefrontal and Anterior Cingulate Cortices. J Neurophysiol 2006;95:2987-3000.

11. Han HB, Lee KE, Choi JH. Functional Dissociation of theta Oscillations in the Frontal and Visual Cortices and Their Long-Range Network during Sustained Attention. eNeuro 2019;6.

12. Fregni F, Simon DK, Wu A, Pascual-Leone A. Non-invasive brain stimulation for Parkinson's disease: a systematic review and meta-analysis of the literature. J Neurol Neurosurg Psychiatry 2005;76:1614-1623.

13. Polania R, Nitsche MA, Ruff CC. Studying and modifying brain function with non-invasive brain stimulation. Nat Neurosci 2018;21:174-187. 
14. Hallett M. Transcranial magnetic stimulation: a primer. Neuron 2007;55:187-199.

15. Ziemann U. Transcranial magnetic stimulation at the interface with other techniques: a powerful tool for studying the human cortex. The Neuroscientist: A Review Journal Bringing Neurobiology, Neurology and Psychiatry 2011;17:368-381.

16. Larson J, Lynch G. Theta pattern stimulation and the induction of LTP: the sequence in which synapses are stimulated determines the degree to which they potentiate. Brain Res 1989;489:49-58.

17. Morgan HM, Jackson MC, van Koningsbruggen MG, Shapiro KL, Linden DE. Frontal and parietal theta burst TMS impairs working memory for visual-spatial conjunctions. Brain Stimul 2013;6:122-129.

18. Benninger DH, Berman BD, Houdayer E, et al. Intermittent theta-burst transcranial magnetic stimulation for treatment of Parkinson disease. Neurology 2011;76:601-609.

19. Xie YJ, Gao Q, He CQ, Bian R. Effect of Repetitive Transcranial Magnetic Stimulation on Gait and Freezing of Gait in Parkinson Disease: A Systematic Review and Meta-analysis. Arch Phys Med Rehabil 2020;101:130-140.

20. Thut G, Miniussi C. New insights into rhythmic brain activity from TMS-EEG studies. Trends Cogn Sci 2009;13:182-189.

21. Rogasch NC, Fitzgerald PB. Assessing cortical network properties using TMS-EEG. Hum Brain Mapp 2013;34:1652-1669.

22. Singh A, Cole RC, Espinoza AI, et al. Timing variability and midfrontal $\sim 4 \mathrm{~Hz}$ rhythms correlate with cognition in Parkinson's disease. NPJ Parkinsons Dis 2021;7:14.

23. Kesar TM, Stinear JW, Wolf SL. The use of transcranial magnetic stimulation to evaluate cortical excitability of lower limb musculature: Challenges and opportunities. Restor Neurol Neurosci 2018;36:333-348.

24. Terao Y, Ugawa Y, Sakai K, Uesaka Y, Kohara N, Kanazawa I. Transcranial stimulation of the leg area of the motor cortex in humans. Acta Neurol Scand 1994;89:378-383.

25. Chipchase L, Schabrun S, Cohen L, et al. A checklist for assessing the methodological quality of studies using transcranial magnetic stimulation to study the motor system: an international consensus study. Clin Neurophysiol 2012;123:1698-1704.

26. Brasil-Neto JP, Cohen LG, Panizza M, Nilsson J, Roth BJ, Hallett M. Optimal focal transcranial magnetic activation of the human motor cortex: effects of coil orientation, shape of the induced current pulse, and stimulus intensity. J Clin Neurophysiol 1992;9:132-136. 
27. Hoeft F, Wu DA, Hernandez A, Glover GH, Shimojo S. Electronically switchable sham transcranial magnetic stimulation (TMS) system. PLoS One 2008;3:e1923.

28. Hoy KE, Bailey N, Michael M, et al. Enhancement of Working Memory and Task-Related Oscillatory Activity Following Intermittent Theta Burst Stimulation in Healthy Controls. Cereb Cortex 2016;26:4563-4573.

29. Nolan H, Whelan R, Reilly RB. FASTER: Fully Automated Statistical Thresholding for EEG artifact Rejection. J Neurosci Methods 2010;192:152-162.

30. Delorme A, Makeig S. EEGLAB: an open source toolbox for analysis of single-trial EEG dynamics including independent component analysis. J Neurosci Methods 2004;134:9-21.

31. Mognon A, Jovicich J, Bruzzone L, Buiatti M. ADJUST: An automatic EEG artifact detector based on the joint use of spatial and temporal features. Psychophysiology 2011;48:229-240.

32. Singh A, Trapp NT, De Corte B, et al. Cerebellar Theta Frequency Transcranial Pulsed Stimulation Increases Frontal Theta Oscillations in Patients with Schizophrenia. The Cerebellum 2019;18:489-499.

33. Cohen MX. Analyzing Neural Time Series Data: Theory and Practice (Issues in Clinical and Cognitive Neuropsychology). The MIT Press; 2014.

34. Noh NA, Fuggetta G, Manganotti P. Theta-burst Transcranial Magnetic Stimulation Alters the Functional Topography of the Cortical Motor Network. The Malaysian Journal of Medical Sciences : MJMS 2015;22:36-44.

35. Klimesch W, Sauseng P, Gerloff C. Enhancing cognitive performance with repetitive transcranial magnetic stimulation at human individual alpha frequency. Eur J Neurosci 2003; $17: 1129-1133$.

36. Klimesch W. EEG alpha and theta oscillations reflect cognitive and memory performance: a review and analysis. Brain Res 1999;29:169-195.

37. Zimerman M, Hummel FC. Non-Invasive Brain Stimulation: Enhancing Motor and Cognitive Functions In Healthy Old Subjects. Front Aging Neurosci 2010;2.

38. Bayot M, Dujardin K, Tard C, et al. The interaction between cognition and motor control: A theoretical framework for dual-task interference effects on posture, gait initiation, gait and turning. Neurophysiol Clin 2018;48:361-375.

39. Wang YK, Jung TP, Lin CT. Theta and Alpha Oscillations in Attentional Interaction during Distracted Driving. Front Behav Neurosci 2018;12:3. 
40. Wagner J, Makeig S, Gola M, Neuper C, Muller-Putz G. Distinct beta Band Oscillatory Networks Subserving Motor and Cognitive Control during Gait Adaptation. J Neurosci 2016;36:2212-2226.

41. Torrecillos F, Tinkhauser G, Fischer P, et al. Modulation of Beta Bursts in the Subthalamic Nucleus Predicts Motor Performance. J Neurosci 2018;38:8905-8917.

42. Doyle LM, Kuhn AA, Hariz M, Kupsch A, Schneider GH, Brown P. Levodopa-induced modulation of subthalamic beta oscillations during self-paced movements in patients with Parkinson's disease. Eur J Neurosci 2005;21:1403-1412.

43. Chung JW, Burciu RG, Ofori E, et al. Beta-band oscillations in the supplementary motor cortex are modulated by levodopa and associated with functional activity in the basal ganglia. Neuroimage Clin 2018;19:559-571.

44. Brown P. Oscillatory nature of human basal ganglia activity: relationship to the pathophysiology of Parkinson's disease. Mov Disord 2003;18:357-363.

45. de Hemptinne C, Swann NC, Ostrem JL, et al. Therapeutic deep brain stimulation reduces cortical phase-amplitude coupling in Parkinson's disease. Nat Neurosci 2015;18:779-786.

46. Whitmer D, de Solages C, Hill B, Yu H, Henderson JM, Bronte-Stewart H. High frequency deep brain stimulation attenuates subthalamic and cortical rhythms in Parkinson's disease. Front Hum Neurosci 2012;6:155.

47. Anidi C, O'Day JJ, Anderson RW, et al. Neuromodulation targets pathological not physiological beta bursts during gait in Parkinson's disease. Neurobiol Dis 2018;120:107-117.

48. Xie T, Vigil J, MacCracken E, et al. Low-frequency stimulation of STN-DBS reduces aspiration and freezing of gait in patients with PD. Neurology 2015;84:415-420.

49. Gilat M, Bell PT, Ehgoetz Martens KA, et al. Dopamine depletion impairs gait automaticity by altering cortico-striatal and cerebellar processing in Parkinson's disease. NeuroImage 2017; 152:207-220.

50. Thut G, Veniero D, Romei V, Miniussi C, Schyns P, Gross J. Rhythmic TMS causes local entrainment of natural oscillatory signatures. Curr Biol 2011;21:1176-1185.

51. Siebner HR, Mentschel C, Auer C, Conrad B. Repetitive transcranial magnetic stimulation has a beneficial effect on bradykinesia in Parkinson's disease. Neuroreport 1999;10:589-594.

52. Samaha J, Gosseries O, Postle BR. Distinct Oscillatory Frequencies Underlie Excitability of Human Occipital and Parietal Cortex. The Journal of Neuroscience 2017;37:2824-2833.

53. Manza P, Hau CL, Leung HC. Alpha power gates relevant information during working memory updating. J Neurosci 2014;34:5998-6002. 
FIGURES
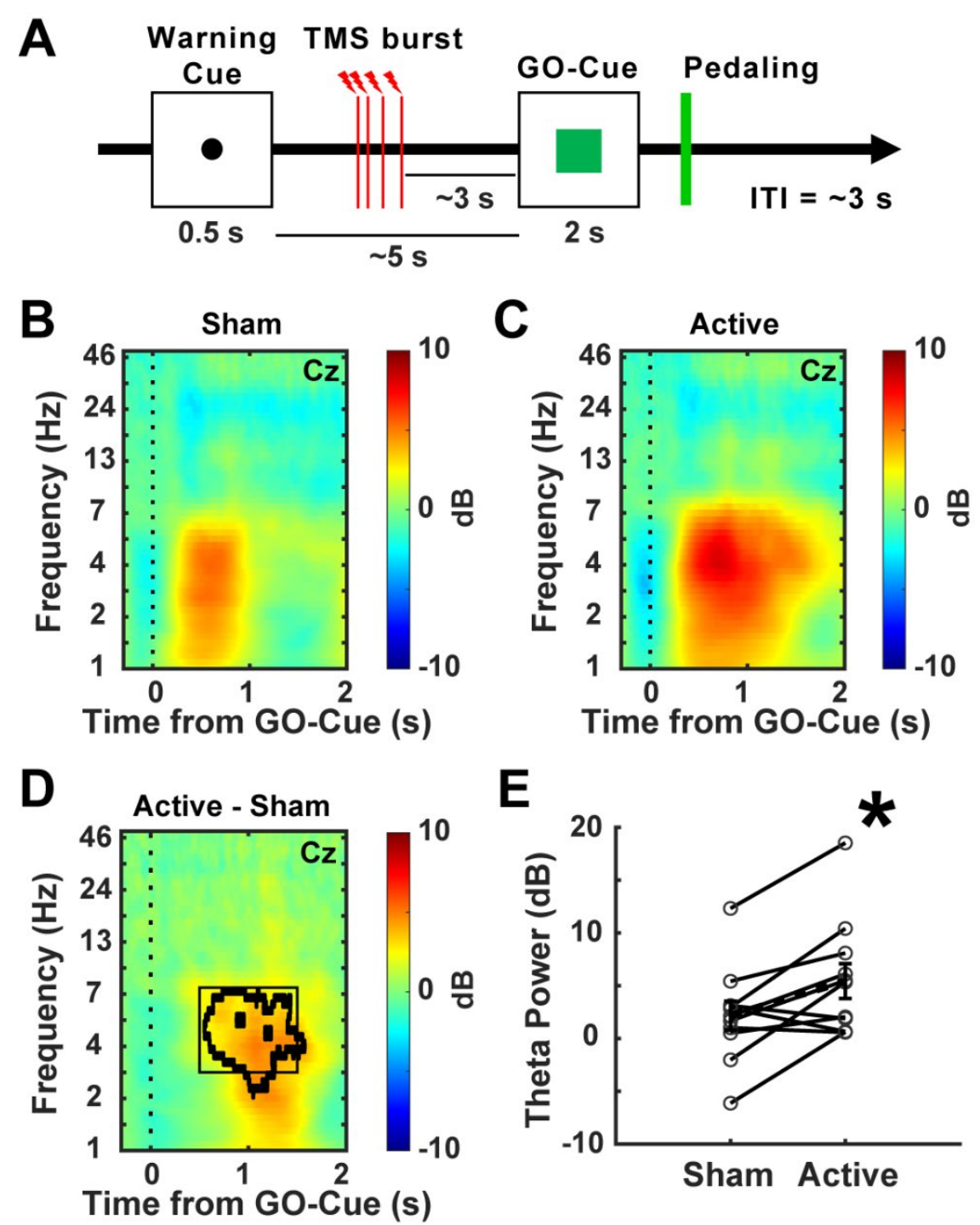

FIG. 1. $\theta$-TMS bursts increased pedaling task-related theta-band activity in the midfrontal region. A) Schematic diagram of the trial of EEG-TMS pedaling task. B-D) Average timefrequency analysis across subjects showed increased theta-band (3-7 Hz) power at midfrontal scalp electrode (vertex or " $\mathrm{Cz}$ ”) during pedaling task after active TMS bursts as compared to sham in healthy elderly subjects. E) Plot displayed the mean power values in tf-ROIs (theta-band power value under black box) during pedaling task. $\mathrm{D}: \mathrm{p}<0.05$ outlined in bold lines. $\mathrm{E}$ : ${ }^{*} \mathrm{p}<0.05$ vs sham stimulation; dashed line with error bar represents the mean and standard error of mean. 

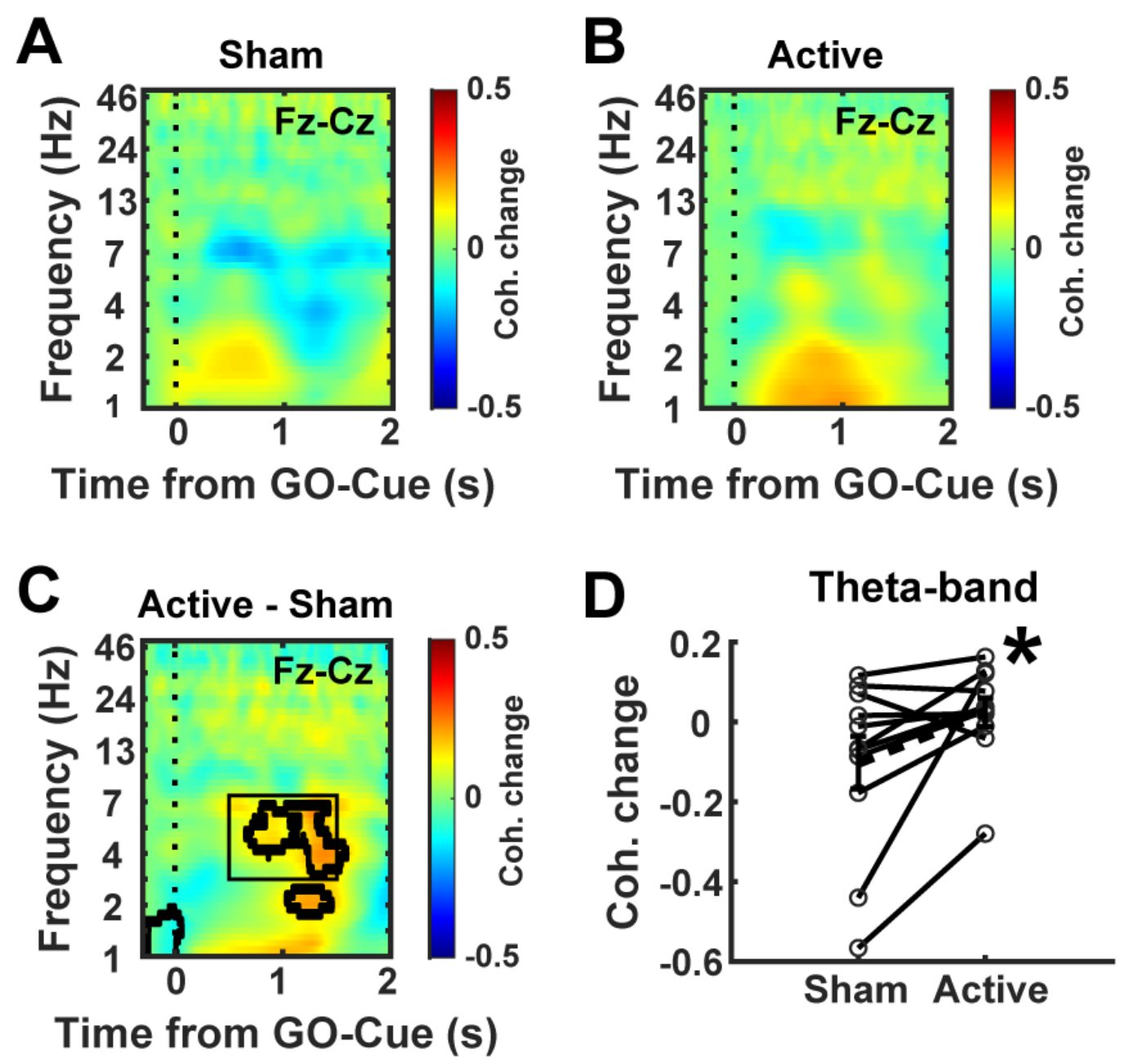

FIG. 2. Spectral coherence in the fronto-cortical network was increased within theta-band.

$\theta$-TMS bursts can increase spectral coherence in the fronto-cortical network $(\mathrm{Fz}-\mathrm{Cz})$ in the theta frequency band during pedaling task in healthy elderly subjects. A) Sham and B) active TMS bursts induced changes in coherence from baseline during pedaling task. C) Difference in coherence between active and sham stimulation. D) Change in coherence from the baseline in the tf-ROIs: theta frequency band during active and sham TMS bursts. C: $p<0.05$ outlined in bold lines and black box represents the $\mathrm{tf}-\mathrm{ROIs}$ in the theta frequency range. $\mathrm{D}:{ }^{*} \mathrm{p}<0.05$; dashed line with error bar represents the mean and standard error of mean. 


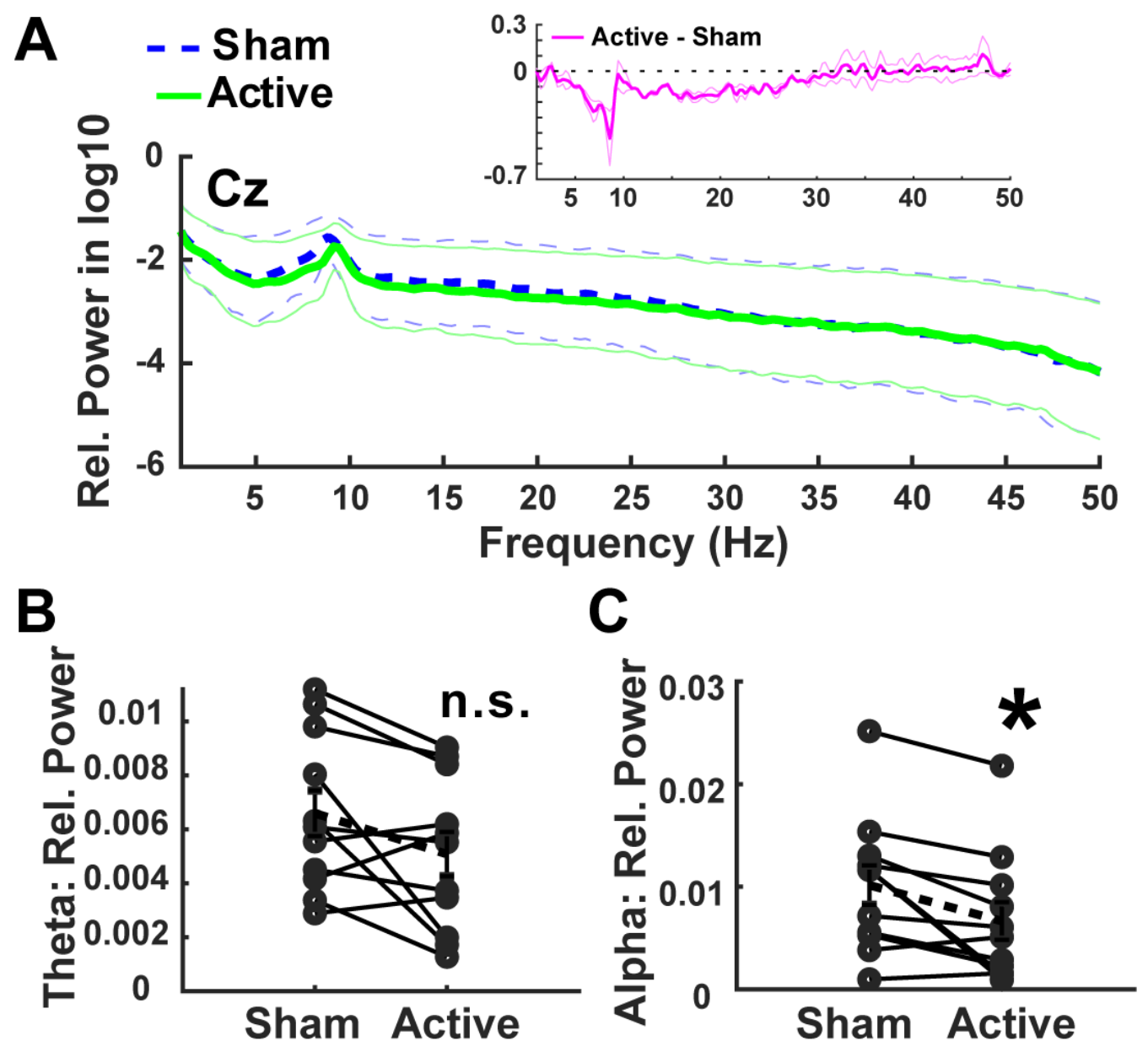

FIG. 3. $\theta$-TMS bursts sessions increased poststimulation resting state midfrontal alpha-band activity. A) Resting-state spectra after task-related active and sham $\theta$-TMS bursts sessions. B-C) Graphs show no difference in theta activity but significant decreases in alpha activity after active TMS bursts sessions. a: thin dashed and solid lines represent the standard error of mean for sham and active stimulation, respectively. ${ }^{*} \mathrm{p}<0.05$ vs. sham; dashed line with error bar represents the mean and standard error of mean; n.s. = non-significant. 


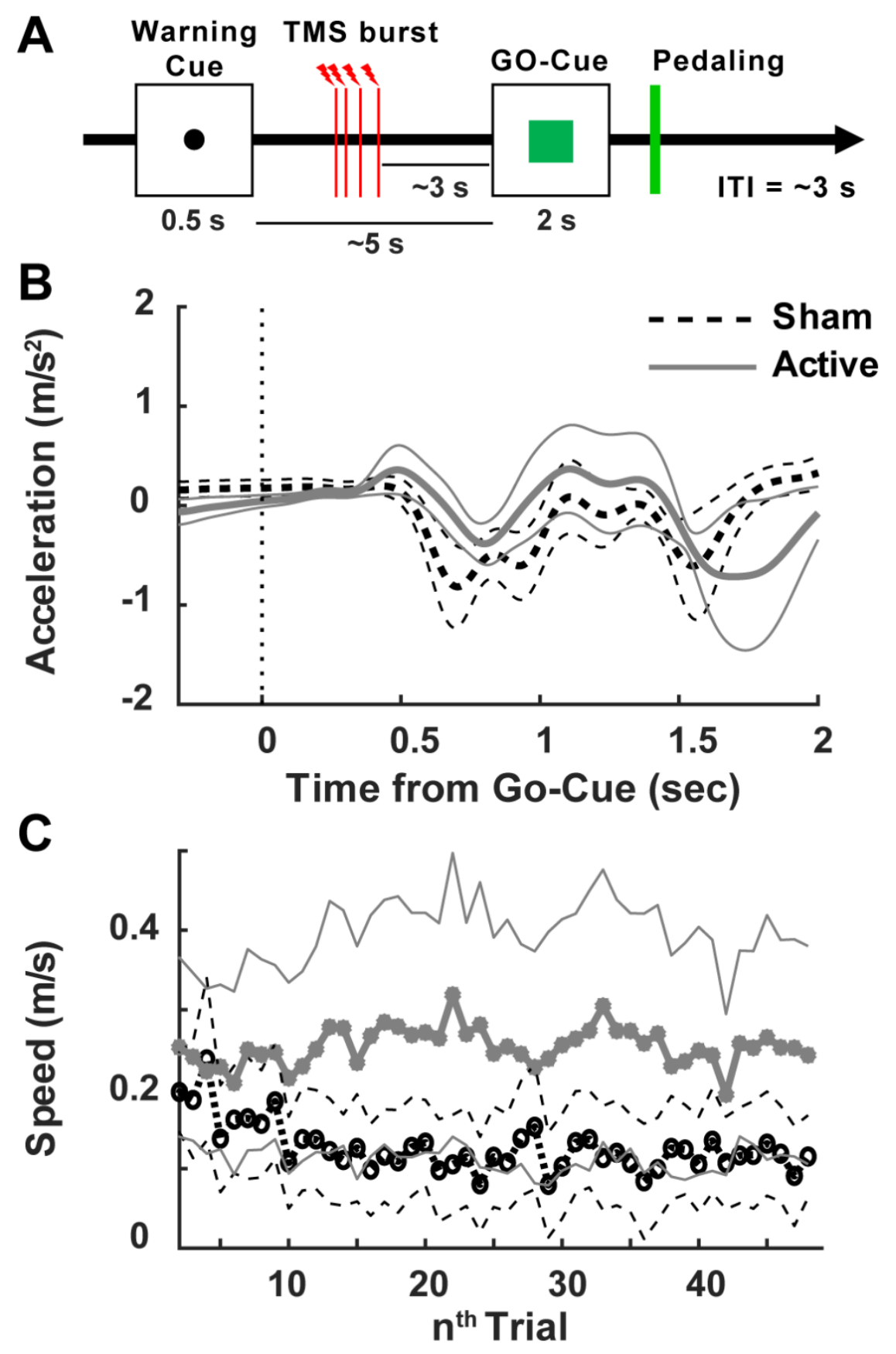

FIG. 4. Effect of $\boldsymbol{\theta}$-TMS bursts on the lower-limb pedaling task. A) Mean acceleration signal after active and sham TMS bursts. B) Time-dependent changes in pedaling speed after active and sham TMS bursts. Thin dashed and solid lines represent the standard error of mean for sham and active stimulation, respectively. 


\title{
SUPPLEMENTARY INFORMATION
}

TMS bursts can modulate local and networks oscillations during lower-limb movement

\author{
Arturo I. Espinoza ${ }^{+, \wedge}$, Jamie L. Scholl ${ }^{*++, \wedge}$, Arun Singh ${ }^{*,++, \#}$ \\ ${ }^{+}$Neurology Department, University of Iowa, Iowa City, IA, USA \\ ${ }^{*}$ Center for Brain and Behavior Research, University of South Dakota, Vermillion, SD, USA \\ ${ }^{++}$Division of Basic Biomedical Sciences, Sanford School of Medicine, University of South \\ Dakota, Vermillion, SD, USA
}




\section{SUPPLEMENTARY FIGURES}

A
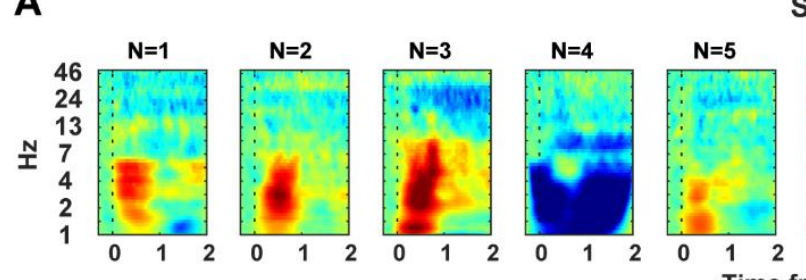

Sham TMS
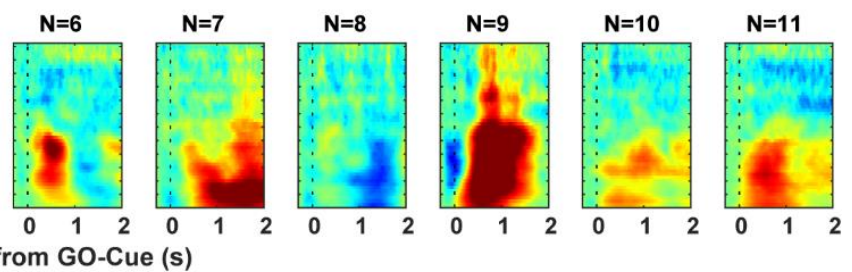

B
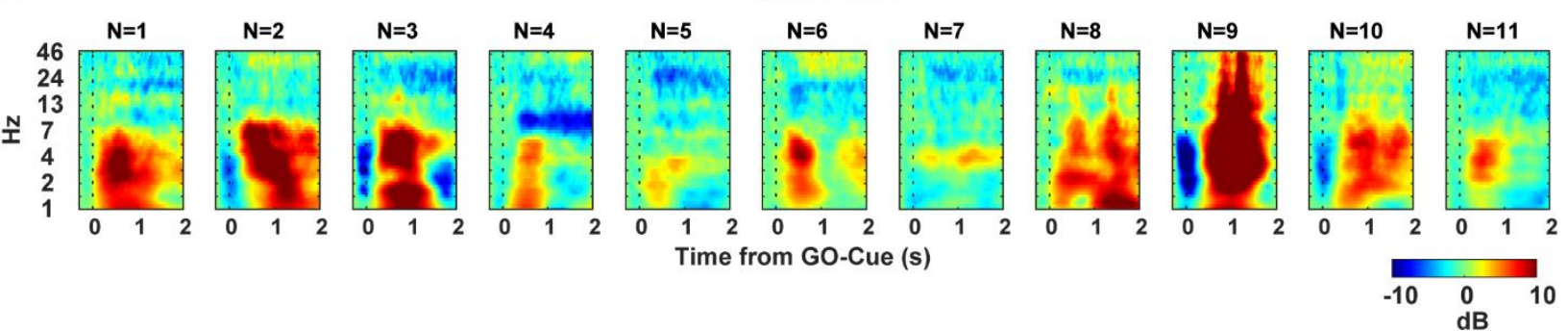

FIG. S1. Active and sham $\theta$-TMS bursts induced midfrontal activity in each subject. a-b)

Pedaling-task related time-frequency plots in the midfrontal lead $(\mathrm{Cz})$ for each healthy elderly subject after sham (A) and active (B) TMS bursts stimulations. 
A

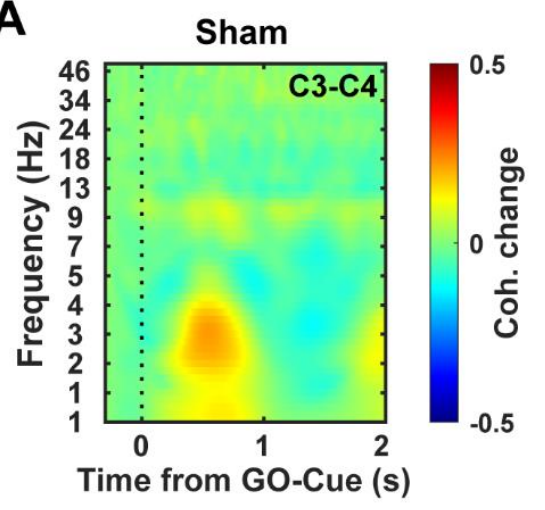

C

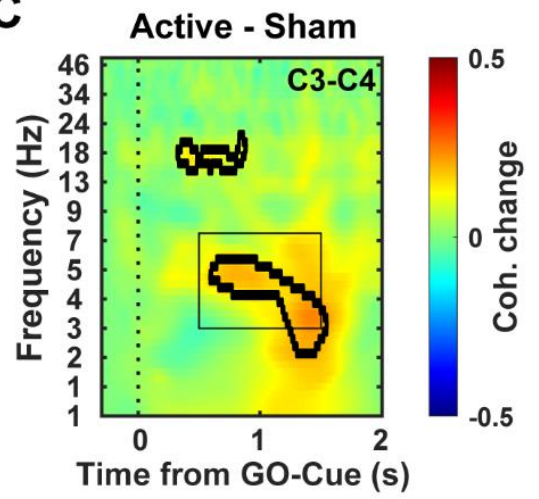

B

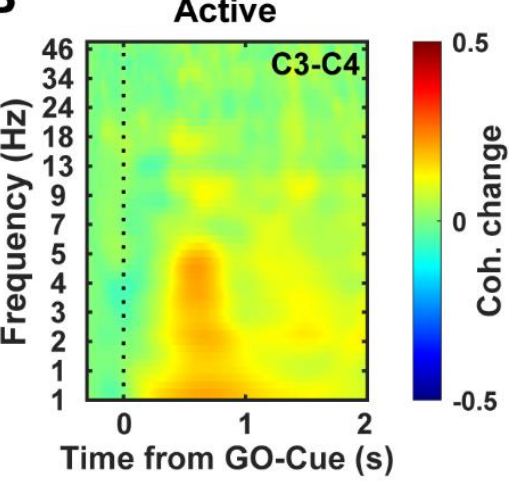

D

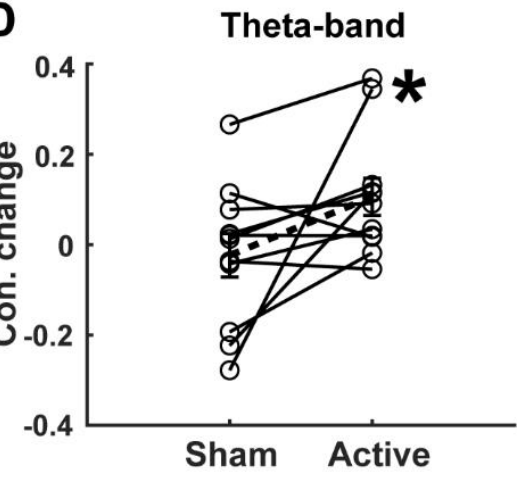

FIG. S2. Spectral coherence in the inter-hemispheric motor cortical network was increased within theta-band. $\theta$-TMS bursts can increase spectral coherence in the inter-hemispheric motor cortical network (C3-C4) within the theta frequency band during pedaling task in healthy elderly subjects. A) Sham and B) active TMS bursts induced changes in coherence from baseline during pedaling task. C) Difference in coherence between active and sham stimulation. D) Change in coherence from the baseline in the tf-ROIs: theta frequency band during active and sham TMS bursts. c: $p<0.05$ outlined in bold lines and black box represents the tf-ROIs in the theta frequency range. d: ${ }^{*} \mathrm{p}<0.05$ vs. sham; dashed line with error bar represents the mean and standard error of mean. 